\title{
Development of bio-hybrid piezoresistive nanocomposites using silk-elastin protein copolymers
}

\author{
Daniela M. Correia ${ }^{\mathrm{a}, \mathrm{b}}$, Sylvie Ribeiro ${ }^{\mathrm{c}, \mathrm{d}}$, André da Costa ${ }^{\mathrm{e}, \mathrm{f}}$, Clarisse Ribeiro $^{\mathrm{c}, \mathrm{g}}$, Margarida Casal ${ }^{\mathrm{e}, \mathrm{f}}$, \\ Senentxu Lanceros-Mendez ${ }^{\mathrm{b}, \mathrm{g}, * *}$, Raul Machado ${ }^{\mathrm{e}, \mathrm{f}, *}$ \\ ${ }^{a}$ Departamento de Química, Universidade de Trás-os-Montes e Alto Douro, 5001-801, Vila Real, Portugal \\ ${ }^{\mathrm{b}}$ BCMaterials, Basque Center for Materials, Applications and Nanostructures, UPV/EHU Science Park, 48940, Leioa, Spain \\ ${ }^{\mathrm{c}}$ Centro de Física, Universidade do Minho, 4710-057, Braga, Portugal \\ ${ }^{\mathrm{d}}$ Centre of Biological Engineering, Universidade do Minho, Campus de Gualtar, 4710-057, Braga, Portugal \\ e CBMA (Centre of Molecular and Environmental Biology), Department of Biology, University of Minho, Campus de Gualtar, 4710-057, Braga, Portugal \\ ${ }_{\mathrm{f}}^{\mathrm{f}}$ IB-S (Institute of Science and Innovation for Sustainability), University of Minho, Campus de Gualtar, 4710-057, Braga, Portugal \\ ${ }^{\mathrm{g}}$ Ikerbasque, Basque Foundation for Science, 48013, Bilbao, Spain
}

\section{A R T I C L E I N F O}

\section{Keywords:}

CNTs

Electromechanical

Gauge factor

Nanocomposites

Piezoresistive

Protein-based

SELP

Silk-elastin-like protein

\begin{abstract}
A B S T R A C T
Recombinant silk-elastin-like protein (SELP)/carbon nanotubes (CNTs) nanocomposite films with different amounts of CNTs (1, 3 and $6 \mathrm{wt} \%)$ were prepared by solvent casting. The produced films were stabilized by exposure to methanol that induces an increase of the $\beta$-structure content. The CNTs were homogeneously distributed into the SELP matrix and did not induce significant alterations into its chemical structure. The incorporation of CNTs also increased the thermal stability of the films. Further, the incorporation of $1 \mathrm{wt} \%$ of CNTs greatly improved the mechanical properties of the SELP matrix leading to a 6-fold increase in strain-to-failure and to increase the ultimate tensile strength with minor differences in modulus of elasticity. The nanocomposites exhibited a good linearity between deformation and electrical resistance variation with electrical conductivity increasing with the nanofiller content up to $0.8 \mathrm{~S} \mathrm{~m}^{-1}$. Finally, the produced nanocomposites were non-cytotoxic indicating their suitability for biomedical applications.
\end{abstract}

\section{Introduction}

Nature offers an abundance of structural proteins with remarkable properties unmatched by known technical materials, covering a wide range of mechanical functions. Fibrous proteins such as collagen, silk and elastin are characterized by repeating blocks of amino acid sequences that result in the formation of well-defined secondary structures which are responsible for the biological properties of the natural protein [1-3]. These repeating sequences have been used as design motifs for the development of recombinant protein-based polymers (rPBPs) displaying properties similar to the natural counterparts [4-6]. Owing to their intrinsic biocompatibility and biodegradability, rPBPs display great potential to be used a platform for the development of biomaterials for biomedical applications [2,7]. For instance, rPBPs have been explored for the controlled release of active agents [8] such as bone morphogenetic proteins [9], as scaffolds for tissue engineering
[2], or for the development of biomaterial blends with improved cell adhesion [10]. Unlike chemical strategies, recombinant DNA technology allows the production of monodisperse polymers with precisely defined sequences. This ability to be fully tailored at the molecular level allows the development of complex multifunctional polypeptides through the combination of distinct peptide domains [11,12]. As a result, the development of genetically engineered protein-based polymers is an emerging field of research that have been the focus of great interest due to the absolute control over chain length and composition, which are key attributes for the design of advanced polymeric materials. This highlights the potential of recombinant protein polymers as central players to revolutionize the use of polymers in materials science.

The silk-elastin-like proteins (SELPs) are a family of such genetically engineered protein polymers combining in the same polypeptide chain the repetitive motifs from Bombyx mori silk and mammalian elastin $[13,14]$. The silk blocks are capable of crystallizing to form physical

\footnotetext{
* Corresponding author. CBMA (Centre of Molecular and Environmental Biology), Department of Biology, University of Minho, Campus de Gualtar, 4710-057, Braga, Portugal.

** Corresponding author. BCMaterials, Basque Center for Materials, Applications and Nanostructures, UPV/EHU Science Park, 48940, Leioa, Spain.

E-mail addresses: senentxu.lanceros@bcmaterials.net (S. Lanceros-Mendez), raulmachado@bio.uminho.pt (R. Machado).
} 
cross-links, providing mechanical stiffness and thermal and chemical stability, while the elastin blocks provide elasticity, decrease the crystallinity and enhances the solubility [13,15]. Due to its versatility of processing and biocompatibility, SELPs have been fabricated into different structures such as hydrogels [16,17], fibres [18,19], films $[15,20]$ and more recently, magnetic nanocomposite films [21], demonstrating unique physical, mechanical and biological properties highlighting its potential for biomedical applications.

The creation of engineered tissues has encouraged the development of new approaches and materials. One of them is the use of composite materials combining synthetic and naturally derived materials due to their potential to provide improved properties with respect to the single material [22,23]. The reinforcement of naturally derived polymers with carbon nanotubes (CNTs) is a promising area leading to the fabrication of polymeric matrices with increased mechanical and electrical properties [22,24]. Furthermore, CNTs have demonstrated their applicability and potential for tissue engineering applications [25]. Therefore, the combination of SELPs and CNTs allows the development of a unique biomaterial with improved and novel characteristics.

In fact, it has been demonstrated that CNTs can be incorporated into different matrices such as collagen, gelatin and silk fibroin for the development of reinforced scaffolds for tissue engineering and regenerative medicine. For instance, collagen/CNT composites incorporating living cells demonstrated to maintain cell viability over a week [22], and composite fibers were able to support fast and efficient neural differentiation [26]. CNTs have been combined with gelatin for the development of multifunctional cardiac scaffolds and bioactuators [27], and with silk fibroin (SF) for the development of nanocomposite fibre mats with increased Young's modulus [28]. Finally, conductive nanocomposite films of SF/CNTs with improved dielectric properties showed to be biocompatible and able to support the growth of dorsal root ganglion neurons [29].

Although displaying interesting properties, SELP films produced by solvent casting are insulating with resistivity values similar to those of natural rubber [15]. In this work, carbon nanotubes have been incorporated in the SELP matrix in order to obtain nanocomposite films endowed with improved piezoresistive response that can find potential use across a wide range of biotechnological areas, including also sensor and actuator applications. A large number of studies report the development piezoresistive sensors based on CNTs with synthetic polymers such as styrene-butadiene-styrene (SBS) [30], poly(vinyl alcohol) (PVA) [31] and polyvinylidene fluoride (PVDF) [32] and also conductive blends such as polyaniline/styrene-butadiene-styrene (PANI/SBS) [33]. However, no studies have previously reported the development of piezoresistive materials based in a semiconductor and genetically engineered protein polymers. This type of materials is increasingly interesting due to its availability to change its electrical resistivity with the application of a mechanical stimulus.

\section{Materials and methods}

\subsection{Materials}

The recombinant silk-elastin-like protein SELP-59-A (SELP, $\mathrm{MW}=56.6 \mathrm{kDa}$ ), was biologically produced and purified as described previously $[14,34]$. The density of the recombinant protein was calculated according to the method proposed by Fischer et al. [35] and determined as $1.413 \mathrm{~g} / \mathrm{cm}^{3}$. The complete amino acid composition [14] consists of 9 tandem repetitions of the sequence S5E9, in which S is the silk-like block with sequence GAGAGS, and $\mathrm{E}$ is the elastin-like block with sequence VPAVG. Multiwall carbon nanotubes (CNTs) with $90 \%$ carbon purity, volume resistivity of $10^{-4} \Omega \mathrm{cm}^{-1}$, average length of $1.5 \mu \mathrm{m}$ and diameter of $9.5 \mathrm{~nm}$ were purchased from Nanocyl (ref. NC7000).

\subsection{Preparation of nanocomposites and post-processing treatment}

Pure lyophilized SELP was directly dissolved in formic acid (98-100\%, Merck) at a concentration of $3 \mathrm{wt} \%$ and mixed thoroughly by vortexing. After complete dissolution, CNTs were added to the protein solution to achieve solutions with 1, 3 and $6 \mathrm{wt} \%$ related to the amount of SELP. The solution was then placed in an ultrasound bath (JP Selecta, nr. 3000617) for $4 \mathrm{~h}$ to obtain a good dispersion. SELP/CNTs composite films were prepared by casting the solution $(7 \mathrm{~mL})$ on bacterial grade polystyrene Petri dishes of $60 \times 15 \mathrm{~mm}$ and the solvent evaporated at room temperature (RT) under extraction. Film thickness was measured using a Dualscope MPOR SK (Fischer Technologies) portable measurement device. The nanocomposite films were stabilized by exposure to methanol (MetOH-treated) at RT for $48 \mathrm{~h}$ in a desiccator [15] and air-dried for at least $48 \mathrm{~h}$ before any characterization.

\subsection{Morphological analysis}

SELP films and SELP/CNTs composite films were transversely cut using liquid nitrogen and subsequently sputter-coated with gold. The samples were visualized and imaged in a Quanta 650 (FEI) field-emission scanning electron microscope (SEM). Cross-sections images were obtained to evaluate the distribution of the CNTs along the sample.

\subsection{Structural characterization}

Attenuated total reflectance-Fourier transform infrared analysis (ATR-FTIR) spectra were acquired from $4000 \mathrm{~cm}^{-1}$ to $400 \mathrm{~cm}^{-1}$ at room temperature with a Spectrum Two spectrometer (Perkin Elmer) coupled with an UATR (single reflection diamond, Perkin Elmer) accessory. Spectra were collected after 64 scans with a resolution of $4 \mathrm{~cm}^{-1}$ with automatic correction of atmospheric $\mathrm{CO}_{2} / \mathrm{H}_{2} \mathrm{O}$. Secondary structure analysis was performed on the amide I band region by second derivative and Gaussian curve fitting [15,36,37] using OriginPro 9.0 software (OriginLab, Northampton, MA). For component analysis, the amide I band region was truncated and normalized, followed by linear baseline correction. The second derivative spectra of the amide I region were smoothed with an eleven-point Savitsky-Golay smoothing function. As data was collected with an interval of $1 \mathrm{~cm}^{-1}$, the selection of an eleven-point window covers an $11 \mathrm{~cm}^{-1}$ spectral region which is less than the half width at half maximum (HWHM) for most of amide I band components [38]. The number of components and peak positions detected by second derivative were used as starting parameters for curve fitting iteratively $\left(\mathrm{R}^{2}>0.999\right)$ with a Gaussian function using the Levenberg-Marquardt algorithm [38,39]. To make the secondary structure assignment comparable between samples, curve fitting was performed with the same set of parameters. Fitting quality was also evaluated by examination of residuals between the original and the fitted curve, and assuming that the extinction coefficient for the $\mathrm{C}=\mathrm{O}$ stretch vibration is the same for the different structural components $[40,41]$. The contribution of each fitted component to the amide I band was determined from the relative area of the single bands by integrating the area under the curve and normalizing for the total area of amide I. Structural conformations were assigned by reference to literature [10,15] (Supplementary Table S1).

\subsection{Thermal properties}

The thermal behavior of the methanol-treated samples was determined by Differential Scanning Calorimetry (DSC) and by ThermoGravimetric Analysis (TGA). DSC measurements were performed with a DSC 6000 Perkin Elmer instrument. For that, small pieces of the sample were randomly cut, placed in aluminum pans and heated from 30 to $250{ }^{\circ} \mathrm{C}$ at a heating rate of $10{ }^{\circ} \mathrm{C} \cdot \mathrm{min}^{-1}$ under a flowing nitrogen atmosphere.

For the TGA measurements, approximately $10 \mathrm{mg}$ of samples were 
transferred to open ceramic crucibles using a TGA 6000 Perkin Elmer instrument operating between $32{ }^{\circ} \mathrm{C}$ to $590{ }^{\circ} \mathrm{C}$ at a heating rate of $10^{\circ} \mathrm{C} \cdot \mathrm{min}^{-1}$ under a flowing nitrogen atmosphere.

\subsection{Mechanical and electromechanical measurements}

Uniaxial tensile failure measurements were carried out at room temperature $\left(25^{\circ} \mathrm{C}\right)$ with a Shimadzu Universal Testing Machine (AG-IS with a load cell of $50 \mathrm{~N}$ ) at a strain rate of $0.5 \mathrm{~mm} / \mathrm{min}$. The ultimate tensile strength (UTS), the strain-to-failure $(\varepsilon)$ and the modulus of elasticity $(E)$ were determined, with the latter being calculated in the linear zone of elasticity, between 0 and $1 \%$ of strain, for all samples.

The conductivity of the samples was evaluated from current (I)voltage (V) curves using an automated Keithley 487 picoammeter/ voltage source. Before analysis, silver electrodes with $5 \mathrm{~mm}$ diameter were painted in the samples. The current intensity was measured while applying voltages between $-0.1 \mathrm{~V}$ and $+0.1 \mathrm{~V}$ (step of $0.01 \mathrm{~V}$ ). The electrical resistance was determined from the slope of the $I-V$ curves and the $d c$ (direct current) conductivity was obtained from equation (1):

$\sigma=1 /(R A / L)$

where in $R A / L$ (electrical resistivity), $R$ is the resistance in Ohm, $A$ is the electrode area in square metres $\left(\mathrm{m}^{2}\right)$, and $L$ is the distance between electrodes in metres. The inverse of the resistivity is the electrical conductivity.

Electromechanical measurements were performed by measuring the electrical resistance (Agilent 34401A multimeter) of the SELP/CNTs composites through silver painted electrodes with simultaneous uniaxial mechanical deformation (Shimadzu AG-IS universal testing machine) with a load cell of $50 \mathrm{~N}$. The experimental measurements were carried out at $1 \mathrm{~mm} / \mathrm{min}$, using rectangular samples with $8 \mathrm{~mm}$ of width and $5 \mathrm{~mm}$ of distance between clamps, and deformations of 0.5 , 1.0, 1.5, 2.0 and $3.0 \%$ with four loading-unloading cycles. The silver painted electrodes were placed inside the clamps to avoid deformation during the uniaxial loading-unloading measurements.

The Gauge Factor (GF) for the composites was calculated using equation (2):

$G F=\frac{d R / R}{d l / L}=\frac{d \rho / \rho}{\varepsilon}+1+2 \nu$

where the applied strain is represented by $\mathrm{dl} / \mathrm{L}=\varepsilon, \rho$ is the electrical resistivity, $\nu$ the Poisson ratio and $(1+2 \nu)$ represent the geometrical contribution to the GF, with a maximum contribution of 2 for elastomers [31]. Because protein networks obey rubber elasticity, a Poisson ratio of 0.5 was used [42].

The critical concentration of the SELP/CNTs composites at which the largest increase of the electrical conductivity occurs was determined by equation (3) according to the percolation theory, with the electrical conductivity near the percolation threshold being characterized by:

$\sigma=\sigma_{0}\left(\varphi-\varphi_{c}\right)^{t}$

where $\sigma_{0}$ and $\varphi$ are the electrical conductivity and volume fraction of the reinforcement material, respectively, $\varphi c$ is the critical volume fraction at the percolation and $t$ is the exponent that correlates with the dimensionality of the system $[43,44]$. Thus, $t$ is the critical exponent related to the dimensionality of the percolative system and is determined from the slope of the $\log (? ?) v s \log \left(\varphi-\varphi_{c}\right)$ plot.

\subsection{In vitro cytotoxicity evaluation}

In vitro cytotoxicity evaluation was assessed with $\mathrm{C} 2 \mathrm{C} 12$ mouse myoblast cell line. Cells were cultured in $75 \mathrm{~cm}^{2}$ cell culture flask at $37^{\circ} \mathrm{C}, 5 \% \mathrm{CO}_{2}$, in humidified environment using Dulbecco's modified Eagles' medium (DMEM, Gibco) containing $4.5 \mathrm{gL}^{-1}$ glucose, $10 \%$ foetal bovine serum (Biochrom) and 1\% (v/v) penicillin/streptomycin solution (Biochrom). Cell viability in response to methanol-treated SELP/CNTs composites was assessed by indirect contact using the MTT (3-(4,5-dimethylthiazol-2-yl)-2,5-diphenyltetrazolium bromide) assay and by following the ISO 10993-12 recommendations [45]. Samples of SELP/CNTs with $6 \mathrm{~cm}^{2}$ were sterilized by multiple immersions into $70 \%$ ethanol for $30 \mathrm{~min}$ each, washed with sterile phosphate-buffered saline solution (PBS; $137 \mathrm{mM} \mathrm{NaCl}, 2.7 \mathrm{mM} \mathrm{KCl}, 10 \mathrm{mM} \mathrm{Na} \mathrm{HPO}_{4}, 1.8 \mathrm{mM}$ $\mathrm{KH}_{2} \mathrm{PO}_{4}$ at $\mathrm{pH}$ 7.4) for $5 \mathrm{~min}$ and exposed to ultraviolet (UV) light for $30 \mathrm{~min}$ each side. The film samples were subsequently incubated with $1 \mathrm{~mL}$ of cell culture medium for $24 \mathrm{~h}$ at $37^{\circ} \mathrm{C}$ with $5 \% \mathrm{CO}_{2}$ in a humidified environment. At the same time, a suspension of $2 \times 10^{4}$ cells $\mathrm{mL}^{-1}$ was seeded in 96 -well tissue culture polystyrene plates for $24 \mathrm{~h}$ under the incubating conditions described above. After this incubation, the cell culture medium was removed and replaced by the as-prepared extraction medium $(100 \mu \mathrm{L})$ conditioned by the $24 \mathrm{~h}$ contact with the film composites. Cell viability was then evaluated after 24 and $72 \mathrm{~h}$ using the MTT proliferation assay according to the manufacturer's (Sigma-Aldrich) instructions. Briefly, MTT solution ( $5 \mathrm{mg} \mathrm{mL}^{-1}$ of MTT dissolved in DMEM in a 1:10 ratio) was added to the cells and incubated in the dark at $37^{\circ} \mathrm{C}$ for $2 \mathrm{~h}$. After the incubation time, the MTT solution was removed and the precipitated formazan was dissolved with $100 \mu \mathrm{L} \mathrm{DMSO} /$ well followed by measuring the absorbance at $570 \mathrm{~nm}$. Cells cultured in standard culture medium and in $20 \%$ DMSO (Sigma-Aldrich) were used as positive and negative controls, respectively. Results were expressed as percentage of viability in relation to the positive control (set as $100 \%$ viability). One-way analysis of variance (ANOVA) with Bonferroni's post-test was carried out using GraphPad Prism 6 software to compare the means of the different data sets within each experiment. A value of $p<0.05$ was considered to be statistically significant. All experiments were performed with at least four replicas.

\section{Results and discussion}

\subsection{Morphology evaluation}

The incorporation of CNTs as nanofillers in polymer matrices leads to the production of composites with tailored electrical, thermal and mechanical properties $[46,47]$. Further, the dispersion of the CNTs within the matrix plays an important role in the determination of these properties. The morphology of the methanol-treated samples with different amounts of CNTs, was analyzed by SEM. Fig. 1 shows representative cross-section SEM images of film samples with 0,1 and $6 \mathrm{wt} \%$ nanofiller content. As demonstrated by Fig. 1a, the SELP film presents a smooth and homogeneous morphology [15]. Fig. 1b-d show a good dispersion of the nanofillers into the protein polymer matrix, even at the highest concentration of $6 \mathrm{wt} \%$. This is also demonstrated at higher magnifications (Fig. 1d) in which the homogenous distribution of the CNTs is confirmed as well as the tubular shape of the CNTs.

\subsection{Structural characterization}

As-cast SELP films are highly water soluble [15] which limits the potential range of applications. The use of methanol has been described as a method to promote structural stability due to a dehydration-induced physical cross-linking, resulting in an increase of the $\beta$-structure content $[15,19,20]$. In this study, the SELP/CNTs nanocomposites were exposed to a methanol-saturated atmosphere and changes in the secondary structure were examined by ATR-FTIR (Fig. 2). Information about the secondary structure was assessed based on the absorption of amide I (mainly $\mathrm{C}=\mathrm{O}$ stretching vibrations) which is a sensitive spectral region used for protein studies. For all cases, the non-treated films display broad absorption bands centred at $1625 \mathrm{~cm}^{-1}$ with a pronounced shoulder at $1644 \mathrm{~cm}^{-1}$ which are indicative of antiparallel $\beta$ sheet and random coil conformations, respectively $[10,15,20]$. This 

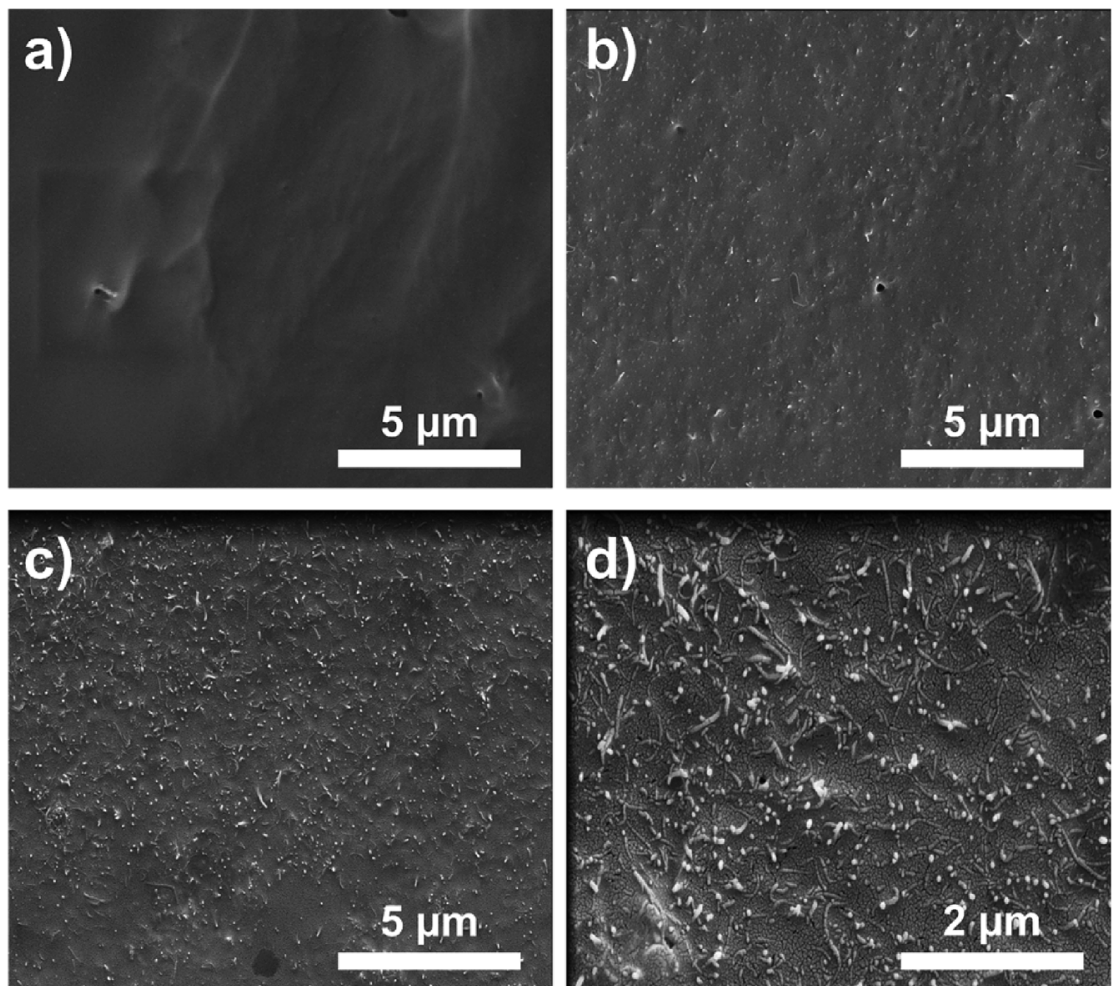

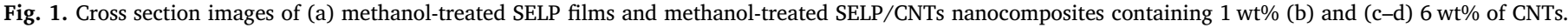

a)

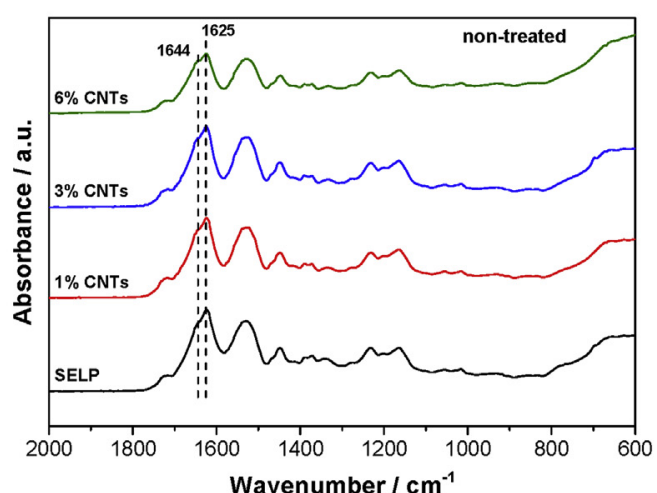

b)

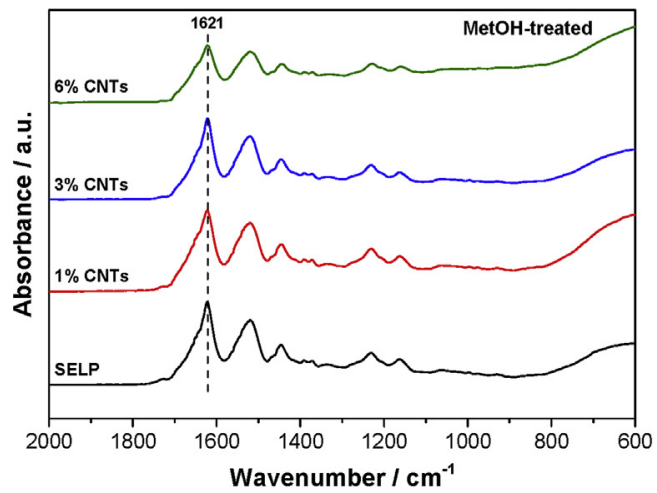

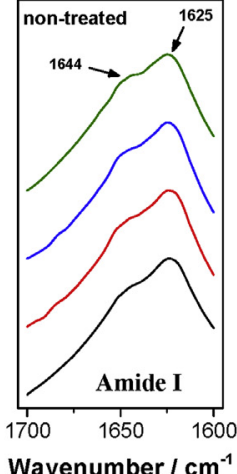

Wavenumber $/ \mathrm{cm}^{-1}$

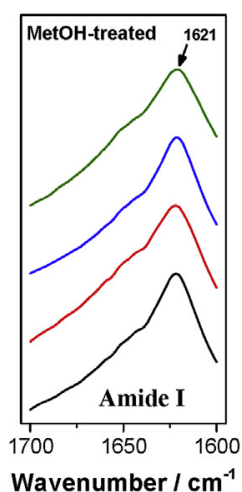

c)

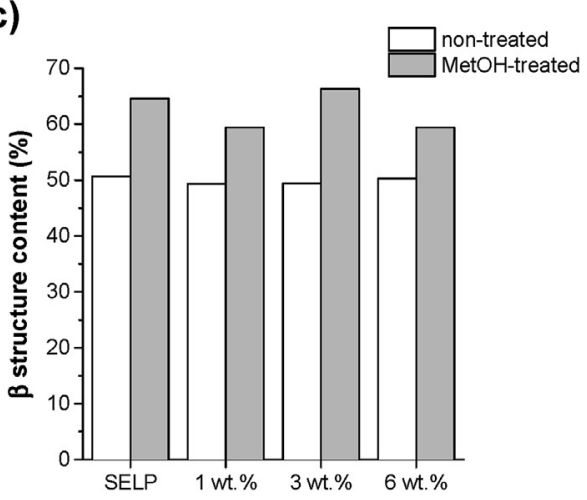

Fig. 2. Attenuated total reflectance Fourier-transform infrared (ATR-FTIR) spectra of (a) non-treated and (b) methanol-treated composites with $0 \mathrm{wt} \%$, $1 \mathrm{wt} \%$, $3 \mathrm{wt} \%$ and $6 \mathrm{wt} \%$ of CNTs. Figure (c) represents the fractional distribution of total $\beta$-structure content determined by second derivative and Gaussian curve fitting. 
a)

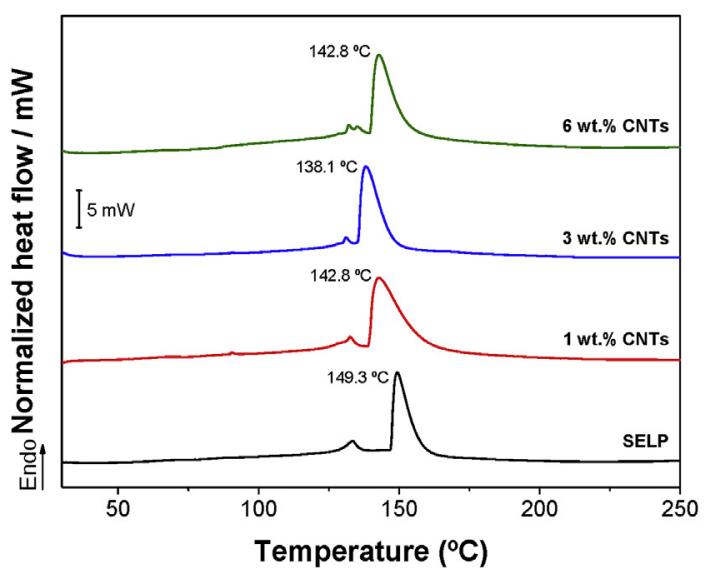

b)

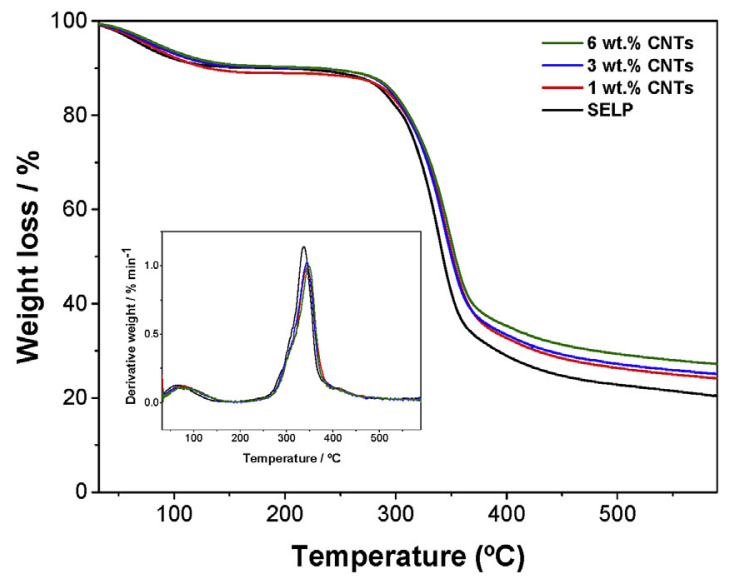

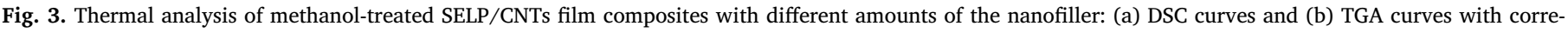
sponding derivative thermogravimetric (DTG) thermograms (inset).

suggests that secondary structure is dominated by $\beta$-sheets with relevant contributions arising from other secondary structures. For all films, after the methanol treatment, the amide I band was narrower and shifted to $1621 \mathrm{~cm}^{-1}$ indicating that major contributions arise from $\beta$ sheet structures and less from other secondary structures. This behavior was previously observed for methanol-treated SELP films $[15,20]$ and SELP composites $[10,21]$ in which treatment with methanol demonstrated to increase the overall content of $\beta$-structures (aggregated strands and $\beta$-sheets). This was further supported by quantitative structural information through the use of second derivative analysis and curve fitting methods in the amide I band region (Fig. S1). After the methanol treatment, the $\beta$-structure content of all films increased from $50 \%$ to approximately $60 \%$ (Fig. 2c). Comparing the IR spectra between pristine SELP and SELP/CNTs nanocomposites, it is observed that the incorporation of the nanofillers do not exerts any chemical alteration or structural changes in the polymer.

\subsection{Thermal characterization}

The thermal properties of the methanol-treated samples with and without CNTs were assessed by DSC and TGA analysis (Fig. 3). Fig. 3a shows the DSC curves for the SELP and SELP/CNTs composites. The thermal events observed in the thermograms are attributed to distinct water states, resulting from water molecules binding to the different film components. All samples display an intense endothermic peak at around $140-150{ }^{\circ} \mathrm{C}$ related with non-freezing bound water present in the films $[48,49]$, and another small endothermic peak that is a consequence of the post-processing treatment with methanol (Table 1). This behavior has been previously observed in magnetic SELP composites and attributed to water entrapped in the more crystalline structure of the methanol treated samples [21]. For the nanocomposites, the incorporation of CNTs in the SELP matrix induces a slight decrease in the temperature of the peak associated to bound water to approximately

Table 1

Overview of the main thermal events of methanol-treated SELP and SELP/CNTs composites determined by DSC and TGA.

\begin{tabular}{llll}
\hline Samples & Endothermic events $\left({ }^{\circ} \mathrm{C}\right)$ & $\begin{array}{l}\text { Decomposition temperature - maximum } \\
\text { rate of weight loss }\left({ }^{\circ} \mathrm{C}\right)\end{array}$ \\
\hline SELP & 134 & 149 & 338 \\
$1 \mathrm{wt} \% \mathrm{CNTs}$ & 133 & 143 & 344 \\
$3 \mathrm{wt} \% \mathrm{CNTs}$ & 131 & 138 & 346 \\
$6 \mathrm{wt} \% \mathrm{CNTs}$ & 132 & 143 & 348 \\
& 135 & & \\
\end{tabular}

$140^{\circ} \mathrm{C}$. This could be a consequence of the good thermal conductivity of the nanofillers that promote a better heat diffusion through the samples. As demonstrated by FTIR (Fig. 2), methanol leads to an increase of the $\beta$-structure content resulting in a more crystalline structure. As a result, the small endothermic peak around $132^{\circ} \mathrm{C}$ is a consequence of the presence of water/moisture in the more closely organized structure of the methanol-treated samples. Interestingly, for the sample with the highest content of CNTs, the thermogram displays an additional small endothermic peak at $135^{\circ} \mathrm{C}$ which can be attributed to the binding of water molecules with the CNTs. The absence of this thermal event at the lowest concentrations of $1 \mathrm{wt} \%$ and $3 \mathrm{wt} \%$ can be explained by the reduced amount of CNTs that are able to interact with water molecules.

The thermal degradation of the methanol-treated film samples was assessed by TGA in order to evaluate their thermal stability. Fig. 3b shows the TGA curves obtained for SELP and SELP/CNTs composites as well as the corresponding derivative thermogravimetric curves. All samples present an initial mass weight loss at around $100{ }^{\circ} \mathrm{C}$ that can be ascribed to the loss of moisture $[15,50]$ due to handling and storage of the samples at room conditions. The major weight loss observed between 250 and $400{ }^{\circ} \mathrm{C}$ corresponds to the effective stage of thermal decomposition and is attributed to the degradation of the material deriving from the breakdown of the side chain amino acid groups and cleavage of the peptide bonds [50,51]. The DTG analysis (inset in Fig. 3b) allows to obtain the temperature of maximum rate of weight loss that is, the decomposition temperature. Accordingly, the maximum point of weight loss is approximately $338^{\circ} \mathrm{C}$ for SELP and above $344^{\circ} \mathrm{C}$ for the SELP/CNTs film composites (Table 1). This demonstrates that the incorporation of CNTs increases the thermal stability of the films, showing a correlation with the nanofiller content: the higher content of nanofiller leads to higher decomposition temperatures (Table 1).

\subsection{Mechanical characterization}

The mechanical properties of the methanol-treated nanocomposites were evaluated under uniaxial tensile strength analysis (Fig. 4). The pure SELP film revealed an average modulus of elasticity $(E)$ of $2.0 \pm 0.8 \mathrm{GPa}$, an average ultimate tensile strength (UTS) of $88 \pm 13 \mathrm{MPa}$ and an average strain-to-failure $(\varepsilon)$ of $7 \pm 4 \%$ (Table 2). These values are within the range of those previously observed for SELP films using the same solvent [10]. Remarkably, the incorporation of $1 \mathrm{wt} \%$ of CNTs demonstrated a significant effect on the mechanical performance. Compared with the SELP film, the composite with $1 \mathrm{wt} \%$ of CNTs presents a much higher ductility, showing an increase of approximately 6-fold in strain-to-failure, and a stress-strain profile characterized by a linear region of elastic behavior and a clear plateau 
a)

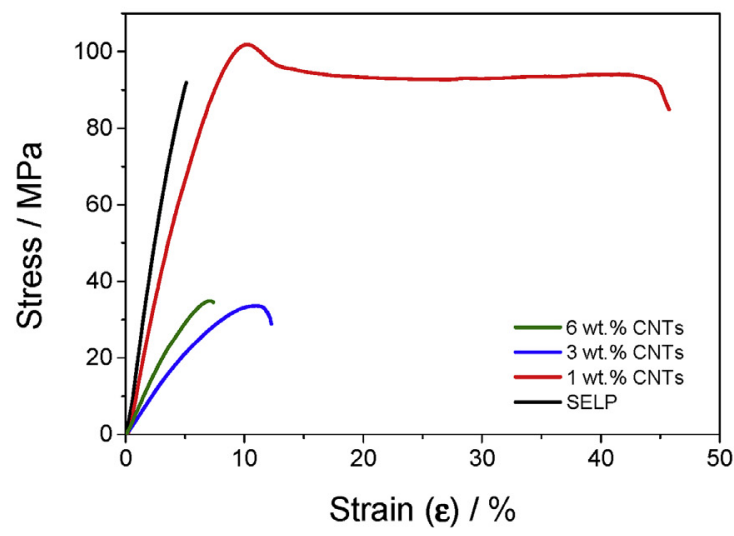

b)

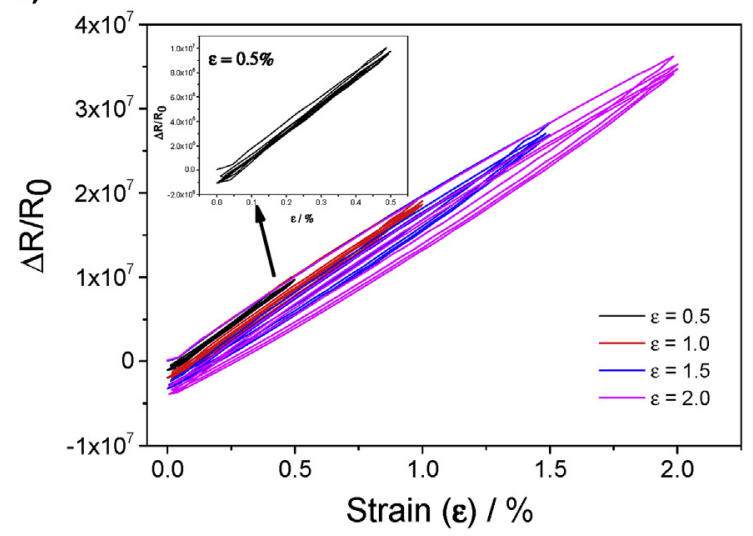

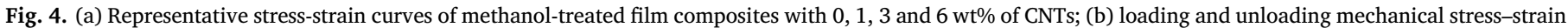
curves for the nanocomposite film with $1 \mathrm{wt} \%$ of CNTs at different strains.

Table 2

Mechanical properties of methanol-treated SELP films incorporating 0,1, 3 and 6 wt \% of CNTs. $E$ - modulus of elasticity; UTS - ultimate tensile strength; $\varepsilon-$ strain-to-failure.

\begin{tabular}{llll}
\hline Sample & $E \pm$ StdDev [MPa] & UTS \pm StdDev [MPa] & $\varepsilon \pm$ StdDev [\%] \\
\hline SELP & $2047 \pm 751$ & $88 \pm 13$ & $7 \pm 4$ \\
$1 \mathrm{wt} \%$ CNTs & $1530 \pm 256$ & $101 \pm 11$ & $43 \pm 17$ \\
$3 \mathrm{wt} \%$ CNTs & $521 \pm 68$ & $34 \pm 6$ & $13 \pm 7$ \\
$6 \mathrm{wt} \% \mathrm{CNTs}$ & $537 \pm 116$ & $34 \pm 20$ & $8 \pm 4$ \\
\hline
\end{tabular}

related to uniform plastic deformation (Fig. 4). In addition to the increased strain-to-failure, with an average value of $43 \pm 17 \%$, the composite with $1 \%$ of CNTs also presents an increased UTS, reaching values of $101 \pm 11 \mathrm{MPa}$ and similar $E$ with a value of $1.5 \pm 0.3 \mathrm{GPa}$ (Table 2). At increased concentrations of $3 \mathrm{wt} \%$ and $6 \mathrm{wt} \%$ it was observed a general decrease of the main mechanical parameters. This suggests that CNTs are providing breaking/failure points that weaken the nanocomposite structure.

Fig. 4b shows the loading-unloading mechanical stress-strain curves for the film with $1 \mathrm{wt} \%$ of CNTs up to a strain of $2 \%$. As observed, the mechanical hysteresis of the films increases with increasing applied strain, demonstrating the increase of the dissipated energy. This behavior has been reported for CNT/elastomer composites [30] and is related to the slow relaxation dynamics of the polymer chains during the unloading cycle.

\subsection{Electrical and electromechanical response}

The electrical conductivity of the nanocomposites as a function of the nanofiller content is presented in Fig. 5. It is known that the conductivity of neat SELP films is around $3.0 \times 10^{-11} \mathrm{~S} \mathrm{~m}^{-1}$ [15]. The incorporation of CNTs into the SELP matrix demonstrated to greatly improve the electrical conductivity with values increasing with the nanofiller content from 0.6 to $0.8 \mathrm{~S} \mathrm{~m}^{-1}$, for the samples with 1 and $6 \mathrm{wt} \%$, respectively. The electrical percolation is observed below $1 \mathrm{wt} \%$ of CNTs and was determined by applying equation (3). According to the fitting of the $\log \left(\varphi-\varphi_{c}\right) v s \log (? ?)$ (see inset of Fig. 5), the percolation threshold of the SELP/CNTs composites is near $\varphi_{c}=0.10 \mathrm{vol} \%$ and $t$ is around 1.45, indicating a quasi-tridimensional CNT percolative system within the SELP matrix $[43,44]$. To notice that the percolation concentration of $0.10 \mathrm{vol} \%(0.5 \mathrm{wt} \%)$ is typical of related systems such as CNT/PVA composites [31].

Regarding the mechanical properties in respect to the percolation concentration, it is observed that the largest decrease of $E$ is observed from the samples containing $1 \mathrm{wt} \% \mathrm{CNTs}$ to the samples containing $3 \mathrm{wt}$

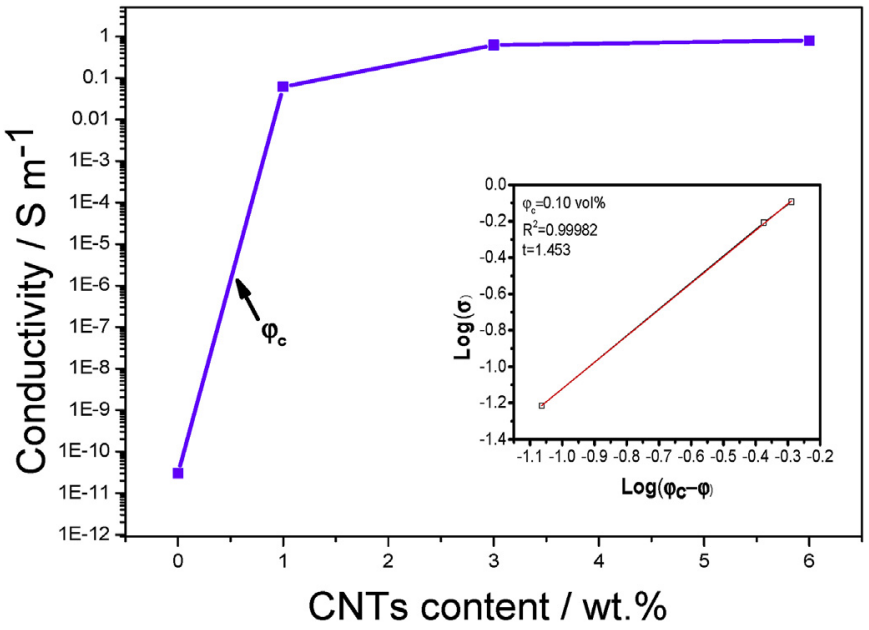

Fig. 5. Electrical conductivity as a function of the CNTs content. Inset image depicts the fitting of the $\log (\varphi-\varphi c)$ vs $\log ($ ??) used for determination of the percolation threshold $\left(\varphi_{c}\right)$.

$\%$. Thus, for concentrations close and higher than $1 \mathrm{wt} \%$, the percolation would start to play an important role, leading to a deterioration of the mechanical properties as result of increasing agglomerates and worst wetting of the CNTs agglomerates by the polymer.

The electromechanical properties of the methanol-treated SELP/ CNTs nanocomposites (Fig. 6) was evaluated during mechanical stress with strains from $0.5 \%$ to $3.0 \%$ and 4 loading-unloading cycles. Fig. 6a-b show a typical example of the electromechanical behavior obtained for the nanocomposite samples with $1 \mathrm{wt} \%$ of CNTs at strains up to $0.5 \%$. Similar results were obtained for the films with 3 and $6 \mathrm{wt} \%$ (results not shown). The samples were characterized by a linear piezoelectric behavior indicating that the electrical resistance follows a direct correlation with the mechanical deformation, increasing linearly upon tensile loading and decreasing linearly upon unloading. The piezoresistive sensitivity of the composites was then quantified by the Gauge Factor (GF) by applying equation (2) (Fig. 6b). The GF of the film composites with $1 \mathrm{wt} \%$ of CNTs demonstrated to be independent of the applied strain, showing values of approximately 0.43 . Accordingly, it is demonstrated that the composites present a piezoresistive behavior characterized by a high reliability performance and sensitivity and are appropriated for biomedical applications or sensors that work at lower deformations. According to equation (2), the GF depends of both the intrinsic piezoresistive effect and the geometric effect, being the contribution of the geometric factor to GF of 2 (Poisson ratio is 0.5 because 

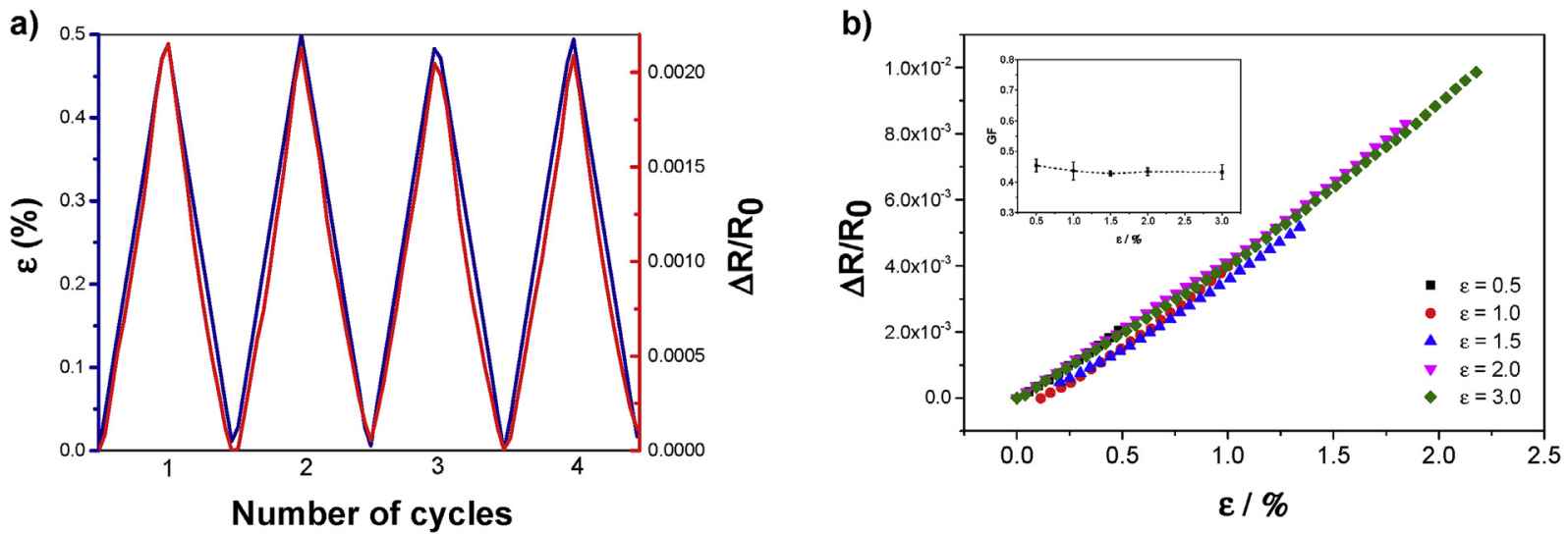

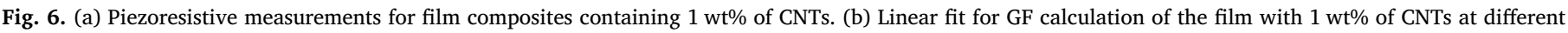
strains $(0.5,1.0,1.5,2.0$ and $3.0 \%)$, with GF as a function of deformation (inset).

protein networks obey rubber elasticity [42]). As the GF values are lower than the geometric factor, independently of the CNTs concentration, the intrinsic piezoresistive effect provides a relevant contribution, opposite to the geometric factor. This fact indicates a reconfiguration of the CNTs network, leading to a decrease in the resistivity with the deformation. Further, it is to notice that the GF is typically larger at the percolation threshold [42], where the percolation network reconfiguration and the associated electrical conductivity variations are larger upon mechanical deformation. Therefore, a slightly lower CNT content will allow to increase the piezoresistive response of the composite.

\subsection{Cytotoxicity assay}

An important parameter to be taken into account in the development of materials for biomedical applications is the absence of cytotoxicity. The cytotoxicity of methanol-treated SELP/CNTs composites was evaluated in vitro by indirect contact and according to ISO 10993 recommendations. The cell viability in response to the methanoltreated films was assessed with $\mathrm{C} 2 \mathrm{C} 12$ mouse myoblast cell lines using the colorimetric MTT assay which gives an indication of cell metabolic activity (Fig. 7). In this assay, metabolically active cells reduce the tetrazolium salts into a formazan dye that can be

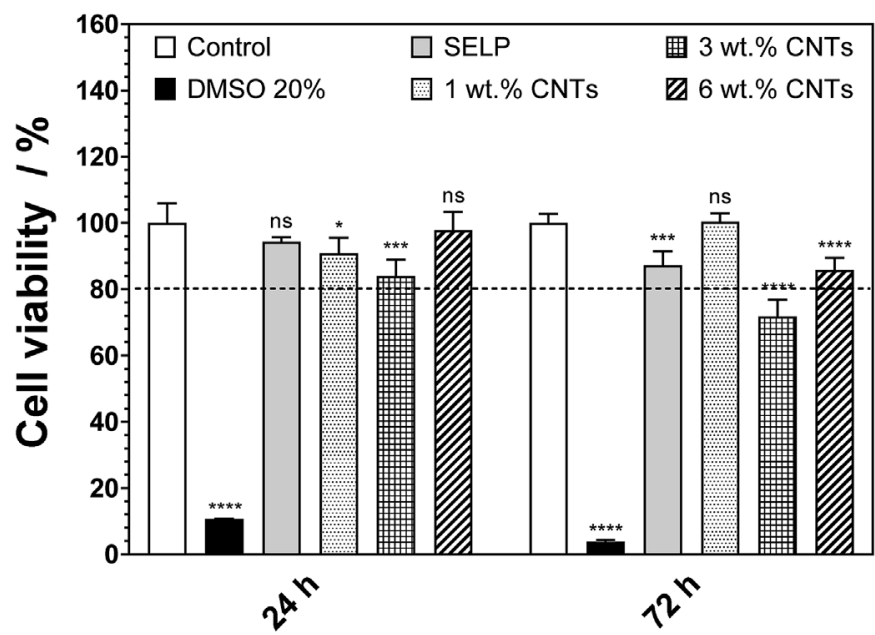

Fig. 7. Cytotoxicity evaluation of methanol-treated SELP/CNTs film composites with different CNTs content ( $0 w t \%, 1 w t \%, 3 w t \%$ and $6 w t \%)$. Cell viability assay was assessed on C2C12 cells using the MTT assay and represented as \% of cell viability in relation to cells cultured in standard medium (positive control). Bars represent mean \pm SD (ns - nonsignificant; *0.01<p<0.05; *** $0.0001<p<0.001 ; * * * * p<0.0001)$. spectrophotometrically measured and is directly related to the number of living cells.

Previously, it was demonstrated that the SELP used in this study namely, SELP-59-A, is non-cytotoxic to normal human skin fibroblasts $[10,19]$. Herein, the same effect is observed for C2C12 cells with the methanol-treated SELP film showing cell viability values of $94.3 \pm 1.4 \%$ and $87.2 \pm 4.3 \%$ after 24 and $72 \mathrm{~h}$, respectively. As for the nanocomposites, all samples display cell viability values above $80 \%$ after $24 \mathrm{~h}$ of exposure, independently of the CNT content. At an increased incubation time of $72 \mathrm{~h}$, it was possible to observe a slight reduction of cell viability for the composites with higher CNT content, reaching values of $71.7 \pm 5.1 \%$ and $85.8 \pm 3.7 \%$ for the samples with $3 \mathrm{wt} \%$ and $6 \mathrm{wt} \%$ of CNTs, respectively. Remarkably, the metabolic activity of cells exposed to leachables of the sample with $1 \mathrm{wt} \%$ of CNTs demonstrated to be similar to the positive control presenting cell viability values of $100.3 \pm 2.7 \%$ even after the $72 \mathrm{~h}$ of incubation.

\section{Conclusions}

The fabrication of piezoresistive SELP/CNTs film composites is demonstrated. The films were fabricated via solvent casting using formic acid as solvent, and further stabilized by exposure to methanol-saturated atmosphere to promote the formation of more $\beta$-structures, rendering water insolubility through a physical crosslinking process. The CNTs are evenly distributed along the protein polymer matrix and increase the thermal stability of the composites without changing the chemical structure. The mechanical properties of the composites with $1 \mathrm{wt} \%$ of CNTs show an increase of 6 -fold in strain-to-failure without significant impact in the strength and modulus of elasticity. The resulting composite materials display an electromechanical response, thus providing evidences of the feasibility of adding piezoresistive functionality to the SELP films. Finally, it is demonstrated that SELP/CNTs composites are non-cytotoxic, indicating its suitability for biomedical applications.

The results of the present work pave the way for the development of a new generation of multifunctional bioinspired materials, opening new perspectives in the future engineering of advanced functional biocomposites.

\section{Acknowledgments}

This work was supported by national funds through FCT I.P. (Fundação para a Ciência e Tecnologia, Portugal) and by the European Regional Development Fund (ERDF) through COMPETE2020 Programa Operacional Competitividade e Internacionalização (POCI, Portugal) in the framework of the Strategic Programs UID/BIA/04050/ 2013 (POCI-01-0145-FEDER-007569) and UID/FIS/04650/2013. This 
work was also supported by the strategic programme UID/BIA/04050/ 2019 funded by national funds through FCT I.P. The present work was also supported by FCT I.P. within the ERA-NET IB-2 project FunBioPlas (ERA-IB-2-6/0004/2014), EuroNanoMed 2016 call and project LungChek ENMed/0049/2016. DMC, SR and CR also thank FCT I.P. for the grants SFRH/BPD/121526/2016, SFRH/BD/111478/2015, and SFRH/BPD/90870/2012, respectively. This article is a result of the project EcoAgriFood [NORTE-01-0145-FEDER-000009], supported by Norte Portugal Regional Operational Programme (NORTE 2020), under the PORTUGAL 2020 Partnership Agreement, through the European Regional Development Fund (ERDF). Finally, the authors acknowledge funding by the Spanish Ministry of Economy and Competitiveness (MINECO) through the project MAT2016-76039-C4-3-R (AEI/FEDER, UE) and from the Basque Government Industry Department under the ELKARTEK and HAZITEK programs.

\section{Appendix A. Supplementary data}

Supplementary data to this article can be found online at https:// doi.org/10.1016/j.compscitech.2019.01.017.

\section{References}

[1] R. Langer, D.A. Tirrell, Designing materials for biology and medicine, Nature 428 (2004) 487-492 https://doi.org/10.1038/nature02388.

[2] M.S. Desai, S.W. Lee, Protein-based functional nanomaterial design for bioengineering applications, Wiley Interdiscip Rev Nanomed Nanobiotechnol 7 (2015) 69-97 https://doi.org/10.1002/wnan.1303.

[3] L.D. Muiznieks, F.W. Keeley, Biomechanical Design of Elastic Protein Biomaterials: A Balance of Protein Structure and Conformational Disorder, ACS Biomater. Sci. Eng. 3 (2017) 661-679 https://10.1021/acsbiomaterials.6b00469.

[4] C. Wong Po Foo, D.L. Kaplan, Genetic engineering of fibrous proteins: spider dragline silk and collagen, Adv. Drug Deliv. Rev. 54 (2002) 1131-1143 https://doi. org/10.1016/S0169-409X(02)00061-3.

[5] D. Olsen, C. Yang, M. Bodo, R. Chang, S. Leigh, J. Baez, D. Carmichael, M. Perala, E.R. Hamalainen, M. Jarvinen, J. Polarek, Recombinant collagen and gelatin for drug delivery, Adv. Drug Deliv. Rev. 55 (2003) 1547-1567 https://doi.org/10. 1016/j.addr.2003.08.008.

[6] W. Teng, J. Cappello, X. Wu, Recombinant silk-elastinlike protein polymer displays elasticity comparable to elastin, Biomacromolecules 10 (2009) 3028-3036 https:// doi.org/10.1021/bm900651g.

[7] J.E. Gagner, W. Kim, E.L. Chaikof, Designing protein-based biomaterials for medical applications, Acta Biomater. 10 (2014) 1542-1557 https://doi.org/10.1016/j. actbio.2013.10.001.

[8] R. Price, A. Poursaid, H. Ghandehari, Controlled release from recombinant polymers, J. Contr. Release 190 (2014) 304-313 https://doi.org/10.1016/j.jconrel. 2014.06.016.

[9] R. Machado, P.C. Bessa, R.L. Reis, J.C. Rodriguez-Cabello, M. Casal, Elastin-based nanoparticles for delivery of bone morphogenetic proteins, 906 (2012) 353-363 https://doi.org/https://10.1007/978-1-61779-953-2_29.

[10] A.M. Pereira, R. Machado, A. da Costa, A. Ribeiro, T. Collins, A.C. Gomes, I.B. Leonor, D.L. Kaplan, R.L. Reis, M. Casal, Silk-based biomaterials functionalized with fibronectin type II promotes cell adhesion, Acta Biomater. 47 (2017) 50-59 https://doi.org/10.1016/j.actbio.2016.10.002.

[11] O.S. Rabotyagova, P. Cebe, D.L. Kaplan, Protein-based block copolymers, Biomacromolecules 12 (2011) 269-289 https://doi.org/10.1021/bm100928x.

[12] R.L. DiMarco, S.C. Heilshorn, Multifunctional Materials through Modular Protein Engineering, Adv. Mater. 24 (2012) 3923-3940 https://doi.org/10.1002/adma. 201200051.

[13] J. Cappello, J. Crissman, M. Dorman, M. Mikolajczak, G. Textor, M. Marquet, F. Ferrari, Genetic engineering of structural protein polymers, Biotechnol. Prog. 6 (1990) 198-202 https://doi.org/10.1021/bp00003a006.

[14] R. Machado, J. Azevedo-Silva, C. Correia, T. Collins, F.J. Arias, J.C. RodriguezCabello, M. Casal, High level expression and facile purification of recombinant silkelastin-like polymers in auto induction shake flask cultures, AMB Express 3 (2013) 11 https://doi.org/10.1186/2191-0855-3-11.

[15] R. Machado, A. da Costa, V. Sencadas, A.M. Pereira, T. Collins, J.C. RodriguezCabello, S. Lanceros-Mendez, M. Casal, Exploring the Properties of Genetically Engineered Silk-Elastin-Like Protein Films, Macromol. Biosci. 15 (2015) 1698-1709 https://doi.org/10.1002/mabi.201500132.

[16] A.A. Dinerman, J. Cappello, H. Ghandehari, S.W. Hoag, Solute diffusion in genetically engineered silk-elastinlike protein polymer hydrogels, J. Contr. Release 82 (2002) 277-287 https://doi.org/10.1016/s0168-3659(02)00134-7.

[17] W.W. Huang, A. Tarakanova, N. Dinjaski, Q. Wang, X.X. Xia, Y. Chen, J.Y. Wong, M.J. Buehler, D.L. Kaplan, Design of Multistimuli Responsive Hydrogels Using Integrated Modeling and Genetically Engineered Silk-Elastin-Like Proteins, Adv. Funct. Mater. 26 (2016) 4113-4123 https://doi.org/10.1002/adfm.201600236.

[18] W. Qiu, Y. Huang, W. Teng, C.M. Cohn, J. Cappello, X. Wu, Complete Recombinant Silk-Elastinlike Protein-Based Tissue Scaffold, Biomacromolecules 11 (2010)
3219-3227 https://doi.org/10.1021/bm100469w.

[19] R. Machado, A. da Costa, V. Sencadas, C. Garcia-Arévalo, C.M. Costa, J. Padrão, A. Gomes, S. Lanceros-Méndez, J.C. Rodríguez-Cabello, M. Casal, Electrospun silkelastin-like fibre mats for tissue engineering applications, Biomed. Mater. 8 (2013) 065009https://doi.org/10.1088/1748-6041/8/6/065009.

[20] L. Chen, M.L. Zhou, Z.G. Qian, D.L. Kaplan, X.X. Xia, Fabrication of Protein Films from Genetically Engineered Silk-Elastin-Like Proteins by Controlled Cross-Linking, ACS Biomater. Sci. Eng. 3 (2017) 335-341 https://10.1021/acsbiomaterials. $6 \mathrm{~b} 00794$.

[21] M.M. Fernandes, D.M. Correia, A. da Costa, S. Ribeiro, M. Casal, S. LancerosMéndez, R. Machado, Multifunctional magnetically responsive biocomposites based on genetically engineered silk-elastin-like protein, Compos. B Eng. 153 (2018) 413-419 https://doi.org/10.1016/j.compositesb.2018.09.019.

[22] R.A. MacDonald, B.F. Laurenzi, G. Viswanathan, P.M. Ajayan, J.P. Stegemann, Collagen-carbon nanotube composite materials as scaffolds in tissue engineering, J. Biomed. Mater. Res. 74 (2005) 489-496 https://doi.org/10.1002/jbm.a.30386.

[23] F.J. O'Brien, Biomaterials \& scaffolds for tissue engineering, Mater. Today 14 (2011) 88-95 https://doi.org/10.1016/S1369-7021(11)70058-X.

[24] S. Ribeiro, P. Costa, C. Ribeiro, V. Sencadas, G. Botelho, S. Lanceros-Méndez, Electrospun styrene-butadiene-styrene elastomer copolymers for tissue engineering applications: Effect of butadiene/styrene ratio, block structure, hydrogenation and carbon nanotube loading on physical properties and cytotoxicity, Compos. B Eng. 67 (2014) 30-38 https://doi.org/10.1016/j.compositesb.2014.06.025.

[25] B.S. Harrison, A. Atala, Carbon nanotube applications for tissue engineering, Biomaterials 28 (2007) 344-353 https://doi.org/10.1016/j.biomaterials.2006.07. 044.

[26] T. Kim, I. Sridharan, B. Zhu, J. Orgel, R. Wang, Effect of CNT on collagen fiber structure, stiffness assembly kinetics and stem cell differentiation, Mater. Sci. Eng. C 49 (2015) 281-289 https://doi.org/10.1016/j.msec.2015.01.014.

[27] S.R. Shin, S.M. Jung, M. Zalabany, K. Kim, P. Zorlutuna, S.B. Kim, M. Nikkhah, M. Khabiry, M. Azize, J. Kong, K.T. Wan, T. Palacios, M.R. Dokmeci, H. Bae, X. Tang, A. Khademhosseini, Carbon-nanotube-embedded hydrogel sheets for engineering cardiac constructs and bioactuators, ACS Nano 7 (2013) 2369-2380 https://doi.org/10.1021/nn305559j.

[28] J. Ayutsede, M. Gandhi, S. Sukiraga, H. Ye, C.M. Hsu, Y. Gogotsi, F. Ko, Carbon nanotube reinforced Bombyx mori silk nanofibers by the electrospinning process, Biomacromolecules 7 (2006) 208-214 https://doi.org/10.1021/bm0505888.

[29] C. Dionigi, T. Posati, V. Benfenati, A. Sagnella, A. Pistone, S. Bonetti, G. Ruani, F. Dinelli, G. Padeletti, R. Zamboni, M. Muccini, A nanostructured conductive biocomposite of silk fibroin-single walled carbon nanotubes, J. Mater. Chem. B 2 (2014) 1424-1431 https://doi.org/10.1039/c3tb21172j.

[30] B.F. Goncalves, P. Costa, J. Oliveira, S. Ribeiro, V. Correia, G. Botelho, S. LancerosMendez, Green Solvent Approach for Printable Large Deformation Thermoplastic Elastomer Based Piezoresistive Sensors and Their Suitability for Biomedical Applications, J. Polym. Sci., Polym. Phys. 54 (2016) 2092-2103 https://doi.org/10 1002/polb.24118.

[31] B.F. Gonçalves, J. Oliveira, P. Costa, V. Correia, P. Martins, G. Botelho, S. Lanceros Mendez, Development of water-based printable piezoresistive sensors for large strain applications, Compos. B Eng. 112 (2017) 344-352 https://doi.org/10.1016/j. compositesb.2016.12.047.

[32] A. Ferreira, V. Correia, E. Mendes, C. Lopes, J.F.V. Vaz, S. Lanceros-Mendez, Piezoresistive Polymer-Based Materials for Real-Time Assessment of the Stump/ Socket Interface Pressure in Lower Limb Amputees, IEEE Sens. J. 17 (2017) 2182-2190 https://doi.org/10.1109/JSEN.2017.2667717.

[33] J. Teixeira, L. Horta-Romarís, M.J. Abad, P. Costa, S. Lanceros-Méndez, Piezoresistive response of extruded polyaniline/(styrene-butadiene-styrene) polymer blends for force and deformation sensors, Mater. Des. 141 (2018) 1-8 https://doi.org/10.1016/j.matdes.2017.12.011.

[34] T. Collins, M. Barroca, F. Branca, J. Padrao, R. Machado, M. Casal, High Level Biosynthesis of a Silk-Elastin-like Protein in E. coli, Biomacromolecules 15 (2014) 2701-2708 https://doi.org/10.1021/bm5005564.

[35] H. Fischer, I. Polikarpov, A.F. Craievich, Average protein density is a molecularweight-dependent function, Protein Sci. 13 (2004) 2825-2828 https://doi.org/10. $1110 /$ ps.04688204.

[36] A. Dong, P. Huang, W.S. Caughey, Protein secondary structures in water from second-derivative amide I infrared spectra, Biochemistry 29 (1990) 3303-3308 https://10.1021/bi00465a022.

[37] P. Taddei, P. Monti, Vibrational infrared conformational studies of model peptides representing the semicrystalline domains of Bombyx mori silk fibroin, Biopolymers 78 (2005) 249-258 https://doi.org/10.1002/bip.20275.

[38] H.Y. Yang, S.N. Yang, J.L. Kong, A.C. Dong, S.N. Yu, Obtaining information about protein secondary structures in aqueous solution using Fourier transform IR spectroscopy, Nat. Protoc. 10 (2015), https://doi.org/10.1038/nprot.2015.024.

[39] J.L. Arrondo, A. Muga, J. Castresana, F.M. Goni, Quantitative studies of the structure of proteins in solution by Fourier-transform infrared spectroscopy, Prog. Biophys. Mol. Biol. 59 (1993) 23-56 https://doi.org/0079-6107(93)90006-6.

[40] C. Jung, Insight into protein structure and protein-ligand recognition by Fourier transform infrared spectroscopy, J. Mol. Recogn. 13 (2000) 325-351 https://doi org/10.1002/1099-1352(200011/12)13:6 <325::AID-JMR507>3.0.CO;2-C.

[41] X. Hu, D. Kaplan, P. Cebe, Determining Beta-Sheet Crystallinity in Fibrous Proteins by Thermal Analysis and Infrared Spectroscopy, Macromolecules 39 (2006) 6161-6170 https://doi.org/10.1021/ma0610109.

[42] X. Hu, X. Wang, J. Rnjak, A.S. Weiss, D.L. Kaplan, Biomaterials derived from silktropoelastin protein systems, Biomaterials 31 (2010) 8121-8131 https://doi.org/ 10.1016/j.biomaterials.2010.07.044.

[43] J.-F. Gao, Z.-M. Li, Q.-j. Meng, Q. Yang, CNTs/UHMWPE composites with a two- 
dimensional conductive network, Mater. Lett. 62 (2008) 3530-3532 https://doi. org/10.1016/j.matlet.2008.03.053.

[44] F. He, S. Lau, H.L. Chan, J. Fan, High Dielectric Permittivity and Low Percolation Threshold in Nanocomposites Based on Poly(vinylidene fluoride) and Exfoliated Graphite Nanoplates, Adv. Mater. 21 (2009) 710-715 https://doi.org/10.1002/ adma.200801758.

[45] R. Machado, V. Sencadas, 7 - Advanced techniques for characterizing bioinspired materials, in: L. Rodrigues, M. Mota (Eds.), Bioinspired Materials for Medical Applications, Woodhead Publishing, 2017, pp. 177-214.

[46] N. Deep, P. Mishra, Evaluation of mechanical properties of functionalized carbon nanotube reinforced PMMA polymer nanocomposite, Karbala Int. J. Mod. Sci. (2018), https://doi.org/10.1016/j.kijoms.2018.02.001.

[47] G. Mittal, V. Dhand, K.Y. Rhee, S.-J. Park, W.R. Lee, A review on carbon nanotubes and graphene as fillers in reinforced polymer nanocomposites, J. Ind. Eng. Chem.
21 (2015) 11-25 https://doi.org/10.1016/j.jiec.2014.03.022

[48] M. Tanaka, T. Hayashi, S. Morita, The roles of water molecules at the biointerface of medical polymers, Polym. J. 45 (2013) 701-710 https://doi.org/10.1038/pj.2012 229.

[49] X. Hu, D. Kaplan, P. Cebe, Effect of water on the thermal properties of silk fibroin, Thermochim. Acta 461 (2007) 137-144 https://doi.org/10.1016/j.tca.2006.12. 011.

[50] A. da Costa, A.M. Pereira, A.C. Gomes, J.C. Rodriguez-Cabello, V. Sencadas, M. Casal, R. Machado, Single step fabrication of antimicrobial fibre mats from a bioengineered protein-based polymer, Biomed. Mater. 12 (2017), https://doi.org/ 10.1088/1748-605X/aa7104.

[51] I.C. Um, H. Kweon, Y.H. Park, S. Hudson, Structural characteristics and properties of the regenerated silk fibroin prepared from formic acid, Int. J. Biol. Macromol. 29 (2001) 91-97 https://doi.org/10.1016/S0141-8130(01)00159-3. 